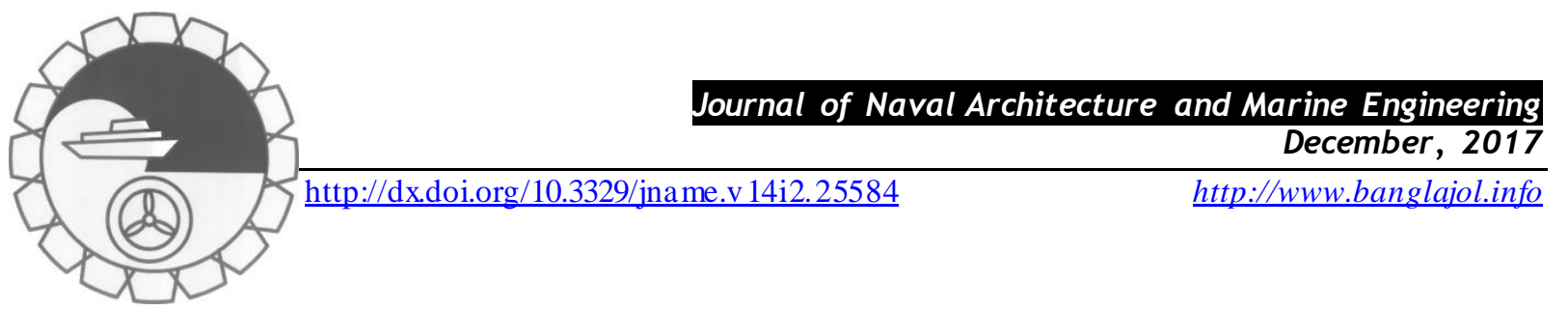

\title{
HEAT AND MASS TRANSFER WITH VISCOUS DISSIPATION IN HORIZONTAL CHANNEL PARTIALLY OCCUPIED BY POROUS MEDIUM IN THE PRESENCE OF OSCILLATORY SUCTION
}

\author{
P. Sharma ${ }^{1}$ and R. Saboo ${ }^{2}$
}

${ }^{1}$ Department of Mathematics and Statistics, Manipal University Jaipur, Jaipur, pooja_2383@yahoo.co.in ${ }^{2}$ Department of Mathematics and Statistics, Manipal University Jaipur, Jaipur, ruchipsaboo@ gmail.com

\begin{abstract}
:
This paper deals with unsteady oscillatory flow of viscous incompressible fluid with heat \& mass transfer in a horizontal channel partially occupied by porous medium following the Darcy-Brinkman model. The inner territory of the channel consists of two regions; one of them is filled with porous material and second is clear fluid. At the porous medium fluid interface, interfacial coupling conditions for the fluid velocity, temperature and concentration were used to derive the analytical solution. Some standard values of Schmidt number like Hydrogen(0.22), Helium(0.30), Amonia(0.78); Eckert number $(0.25,0.5,1,2)$ and Prandtl number for water(0.71) and Air(7.0) was considered in this paper. The effects of pertinent physical fluid parameter like porosity, viscosity ratio, density ratio etc. on velocity, temperature and concentration distribution are considered and demonstrated through graphs. Heavier diffusive species and high molecular diffusivity ratio decreases the fluid concentration throughout the region. Increasing values of Prandtl number upsurge the fluid temperature throughout the region.
\end{abstract}

Keywords: Oscillatory flow, horizontal channel, viscous dissipation, mass transfer.

\section{NOMENCLATURE}

$C_{i}^{*} \quad$ Species concentration

$t^{*} \quad$ Time

$u_{i}^{*} \quad$ Velocity component along x-axis

$T_{w_{1}}^{*} \quad$ Temperature of the upperplate

$K_{p}^{*} \quad$ Permeability of the medium

$T_{i}^{*} \quad$ Temperature of the fluid

$T^{*} \quad$ Temperature of the lower plate

$v_{* 2}^{W_{2}}$ Suction velocity

$P \quad$ Non-dimensional pressure gradient

$\Delta T \quad$ Temperature gradient

$m \quad$ Ratio of viscosities of the fluids

D Molecular Diffusivity ratio

$y \quad$ Non-dimensional distance along y-axis

Greek symbols

$\begin{array}{ll}\omega^{*} & \text { Frequency parameter } \\ \omega & \text { Non-dimensional frequency of oscillation } \\ \kappa_{i} & \text { Thermal conductivity }\end{array}$

$\begin{array}{ll}C_{w_{1}}^{*} & \begin{array}{l}\text { Concentration of the species at the upper } \\ E c\end{array} \\ N u & \text { Eckert number } \\ & \text { Nusselt number } \\ \operatorname{Pr} & \text { Prandtl number } \\ & \text { Ratio of coefficient of volumetric thermal } \\ n & \text { expansion of the fluid for mass transfer } \\ \mathrm{D}_{\mathrm{i}} & \text { Molecular diffusivity of the fluid } \\ A & \text { Real positive constant } \\ C & \text { Skin friction coefficient } \\ G c & \text { Grashof number for mass transfer } \\ K & \text { Porous medium parameter } \\ \Delta C & \text { Species concentration gradient } \\ U_{0} & \text { Average velocity } \\ g & \text { Acceleration due to gravity }\end{array}$

$\theta_{i} \quad$ Dimensionless temperature of the fluid

$v_{0} \quad$ Mean velocity

Unematic viscosity 


\begin{tabular}{llll}
\hline$\mu_{i}$ & Viscosity of the fluid & $\eta$ & Density ratio \\
$\rho_{i}$ & Density of the fluid & $\in$ & A small positive constant \\
$\beta f_{i}$ & $\begin{array}{l}\text { Volumetric coefficient of expansion with heat } \\
\text { transfer }\end{array}$ & $\beta_{c_{i}}$ & $\begin{array}{l}\text { Volumetric coefficient of expansion with } \\
\text { mass transfer }\end{array}$ \\
$\omega t$ & $\begin{array}{l}\text { Periodic frequency parameter } \\
\text { nan }\end{array}$
\end{tabular}

Subscripts $\hat{i}=1,2$ quantities for region-I and region-II respectively.

\section{Introduction}

The study of fluid flow problems related with heat and mass transfer is of widespread interest in almost all the fields of engineering as well as in astrophysics, biology, biomedicine, meteorology, physical chemistry, plasma physics, geophysics, oceanography and scores of other disciplines. Heat and mass transfer in porous media has attracted considerable attention due to the wide range of thermal engineering applications as geothermal systems, oil extraction, ground water pollution, thermal insulation and solid matrix heat exchangers etc. Improvement of heat transfer in thermal devices such as heat exchangers and electronic devices has become an important factor in various industries. For this purpose, various techniques have been proposed as the use of fins, baffles, blocks and porous media. Heat and mass transfer on unsteady flow in a horizon tal porous channel filled with porous material has gained interest owing to its application in the field of gas turbines, the various propulsion devices for aircraft, missiles, satellites and space vehicles.

In respect of above, fluid flow and convective heat and mass transfer in channels partially filled by porous medium have been discussed by several investigators analytically, experimentally and numerically. Kuznetsov(2001) studied analytically the effect of thermal dispersion on fully developed forced convection flow in a parallel-plate channel partly filled with a saturated porous medium. In the geometry, peripheral parts of the channel were occupied by a fluid-saturated porous medium of uniform porosity. Nield et al.(2004) discussed the effects of viscous dissipation and flow work on forced convection in a channel filled by saturated porous medium. In continuation of that Umavathi et al.(2005) considered a two-dimensional unsteady flow of two immiscible fluids through a horizontal parallel permeable plates, and they concluded that the flow and heat transfer aspects in a horizontal channel with permeable walls can be controlled by considering different fluids having different viscosities, conductivities and also by varying the amplitude of the transpiration velocity at the boundary. Bakkas et al.(2008) illustrated natural convection flow in horizontal channel provided with rectangular blocks releasing uniform heat flux and mounted on its lower wall. Umavathi et al.(2009) studied the unsteady oscillatory flow and heat transfer in a horizontal composite porous medium channel. Kuznetsov et al.(2010) studied the forced convection in a channel partly occupied by bidisperse porous medium. Chen et al.(2011) found exact analytical solution to a fully developed forced convection heat transfer in a parallel plate channel. They concluded that effect of viscous dissipation is significant on heat transfer inside channels. Sharma et al.(2011) studied the influence of radiation on unsteady MHD free convective flow and mass transfer through viscous fluid past a heated vertical plate immersed in porous medium.

Problem involving multiphase flow, heat transfer and multi-component mass transfer arise in a number of scientific and engineering disciplines and are important in the petroleum extraction and transport. Therefore several authors have been done some theoretical and experimental work on fluid flow through porous or nonporous parallel plates. Kumar et al.(2012) studied the unsteady MHD free convection flow through porous medium sandwiched between viscous fluids in a horizontal channel with is othermal walls temperature, has been investigated using Brinkman model. They concluded that the fluid velocity props up with an increase of the ratio of Grashof number to the Reynolds number, thermal conductivity ratio, whereas the fluid temperature increases with the increase of the thermal conductivity ratio. Ghachem et al.(2012) presented numerical simulation of three-dimensional double diffusive natural convection flow and irreversibility studies in a solar distiller. Matin et al.(2013) analyzed the effects of chemical reaction in the flow through porous channel with a first order chemical reaction on the wall. They studied the fully developed forced convection flow and mass trans fer in a horizontal porous channel filled by nano fluid. Hussein et al.(2013) discussed free convection in a square enclosure filled with a saturated porous matrix under different discrete heat source locations.

Ahmed et al.(2014) studied effects if thermal radiation viscous dissipation on MHD free convection in a square enclosure filled with a porous medium. Adegun et al.(2014) found three-dimensional finite element 
investigation of incompressible fluid flow and contaminant transport through a porous landfill. Raju et al.(2014) analytically studied MHD convective flow through porous medium in a horizontal channel with insulated and impermeable bottom wall in the presence of viscous dissipation and joule heating. In which the governing equations are solved in the closed form and mean velocity, mean temperature, mean mixed temperature is also considered in this study. Then Choudhary et al.(2015) presented transient MHD visco-elastic flow past an impulsively started infinite porous plate in the presence of hall current. Maatki et al.(2016) discussed effects of inclination of intensity magnetic field in three -dimensional double diffusive free convection flow. Recently, Sharma et al.(2017) studied theoretical study of heat and mass transfer in the presence of chemically reacting radiating MHD flow through horizontal channel, in which they concluded that for high depth porous medium channel the fluid mean temperature and species mean concentration both rise high. Fluid flow can be made fast in this geometrical fluid flow by increasing the depth of the porous medium. Reddy Et al.(2017) investigated dual solutions for heat and mass transfer in chemically reacting radiative non-newtonian fluid.

Inspired by the above citied research work the aim of the present paper is to investigate the effects mass transfer in forced convective viscous flow through porous medium. Above referenced work and the numerous possible industrial applications of the problem, an attempt has been made to study the heat and mass transfer in unsteady oscillatory flow through horizontal channel partially occupied by porous medium one of them is filed with porous material and other one is clear fluid. The flow is modeled using the Darcy-Brinkman equation. The viscous and darcian terms are also included in the energy equation.

\section{Mathematical Formulation}

Consider unsteady fully developed, laminar flow of an incompressible viscous fluid through an infinitely -long composite channel, consisting of two parallel permeable plates with half of the distance between them filled by a fluid-saturated porous layer and the other half by a clear viscous fluid.

A schematic diagram of the problem is shown in figure-1. The x-axis is taken parallel to the axis of the channel while the $y$-axis is taken normal to it. Where $2 \mathrm{~h}$ is the height of the channel

Two horizontal parallel plates are set in the plane $y=h$ (Region-I) and $y=-h$ (Region-II) and kept at constant temperature $\quad T_{w_{1}}, T_{w_{2}}$ and constant species concentration $C_{w_{1}}, C_{w_{2}}$ respectively, with $T_{w_{2}}<T_{w_{1}}$ and $C_{w_{2}}<C_{w_{1}}$. The region $0<y<h$ (region I) is occupied by a clear viscous fluid and the region $-h<y<0$ (region II) is filled with a porous matrix.

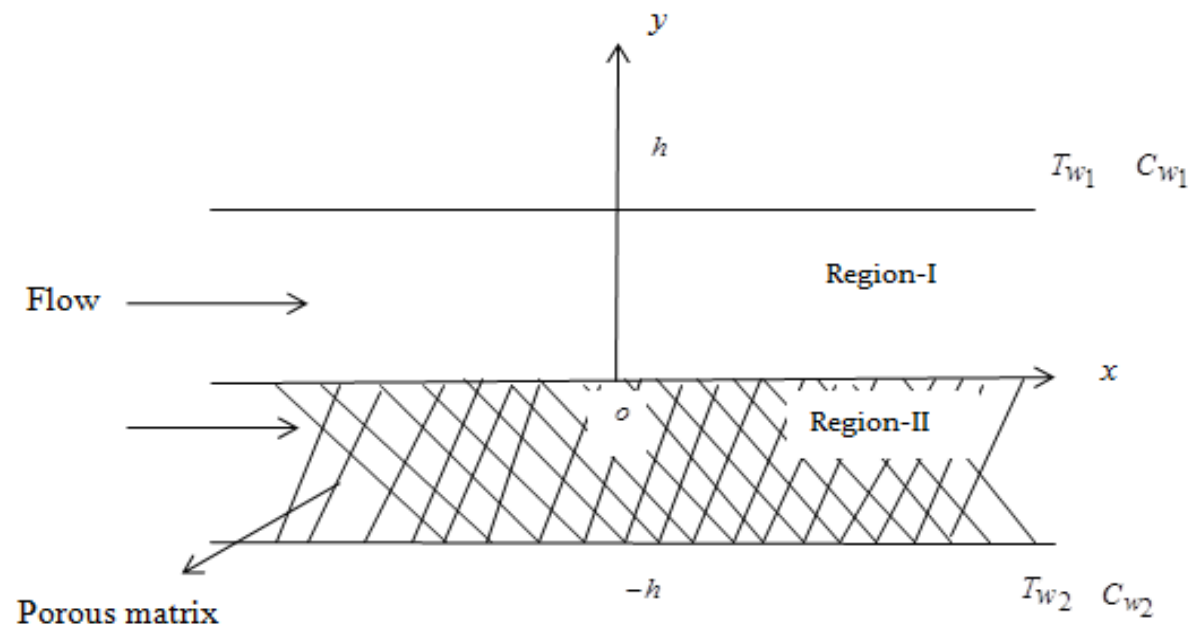

Fig. 1: Geometrical configuration

Since the plates of the channel are assumed to be infinite. Therefore the entire dependent physical variable except pressure will only depend on y and t. The thermo-physical properties of the fluid and effective properties of the porous medium are supposed to be constant. Fluid and porous matrix are supposed to be in local thermal equilibrium in region-II. Further the flow in both regions of channel is expected to be driven by a common pressure gradient $(-\partial P / \partial x)$, temperature gradient $\Delta T=T_{w_{2}}-T_{w_{1}}$ and concentration gradient 
$\Delta C=C_{w_{2}}-C_{w_{1}}$. The flow in the channel follows the Darcy-Brinkman model. Viscous and darcian dissipation effects are taken into account in the energy equation.

Under these assumptions, there are two sets of governing equations corresponding to the porous region and clear fluid region as follows:

Region-I Clear Region

$$
\begin{aligned}
& \frac{\partial v_{1}^{*}}{\partial y^{*}}=0, \\
& \rho_{1}\left(\frac{\partial u_{1}^{*}}{\partial t^{*}}+v_{1}^{*} \frac{\partial u_{1}^{*}}{\partial y^{*}}\right)=\mu_{1} \frac{\partial^{2} u_{1}^{*}}{\partial y^{* 2}}-\frac{\partial p^{*}}{\partial x^{*}}+\rho_{1} g \beta_{c_{1}}\left(C_{1}^{*}-C_{w_{1}}^{*}\right), \\
& \rho_{1} C_{p}\left(\frac{\partial T_{1}^{*}}{\partial t^{*}}+v_{1}^{*} \frac{\partial T_{1}^{*}}{\partial y^{*}}\right)=\kappa_{1} \frac{\partial^{2} T_{1}^{*}}{\partial y^{* 2}}+\mu_{1}\left(\frac{\partial u_{1}^{*}}{\partial y^{*}}\right)^{2}, \\
& \left(\frac{\partial C_{1}^{*}}{\partial t^{*}}+v_{1}^{*} \frac{\partial C_{1}^{*}}{\partial y^{*}}\right)=D_{1} \frac{\partial^{2} C_{1}^{*}}{\partial y^{* 2}},
\end{aligned}
$$

Region-II: Porous Region

$$
\begin{aligned}
& \frac{\partial v_{2}^{*}}{\partial y^{*}}=0, \\
& \rho_{2}\left(\frac{\partial u_{2}^{*}}{\partial t^{*}}+v_{2}^{*} \frac{\partial u_{2}^{*}}{\partial y^{*}}\right)=\mu_{2} \frac{\partial^{2} u_{2}^{*}}{\partial y^{* 2}}-\frac{\partial p^{*}}{\partial x^{*}}-\frac{\mu_{2}}{K_{p}^{*}} u_{2}^{*}+\rho_{2} g \beta_{c_{2}}\left(C_{2}^{*}-C_{w_{1}}^{*}\right), \\
& \rho_{2} C_{p}\left(\frac{\partial T_{2}^{*}}{\partial t^{*}}+v_{2}^{*} \frac{\partial T_{2}^{*}}{\partial y^{*}}\right)=\kappa_{2} \frac{\partial^{2} T_{2}^{*}}{\partial y^{* 2}}+\mu_{2}\left(\frac{\partial u_{2}^{*}}{\partial y^{*}}\right)^{2}+\frac{\mu_{2}}{K_{p}^{*}} u_{2}^{* 2}, \\
& \left(\frac{\partial C_{2}^{*}}{\partial t^{*}}+v_{2}^{*} \frac{\partial C_{2}^{*}}{\partial y^{*}}\right)=D_{2} \frac{\partial^{2} C_{2}^{*}}{\partial y^{* 2}},
\end{aligned}
$$

The boundary conditions and interface condition on velocity are the no-slip boundary conditions because the fluid particles at the boundary and interface come at rest, which require that the x-components of velocity to be vanished at the wall. The boundary conditions on temperature are followed by the isothermal conditions. Then hydrodynamic boundary and interface conditions for velocity of two fluids can be written as

$u_{1}^{*}(h)=0, \quad u_{2}^{*}(-h)=0$,

$u_{1}^{*}(0)=u_{2}^{*}(0)$,

$\mu_{1} \frac{\partial u_{1}^{*}}{\partial y^{*}}=\mu_{2} \frac{\partial u_{2}^{*}}{\partial y^{*}} \quad$ at $y^{*}=0$.

The boundary and the interface conditions on temperature for both the fluids are given by

$T_{1}^{*}(h)=T_{w_{1}}, \quad T_{2}^{*}(-h)=T_{w_{2}}$,

$T_{1}^{*}(0)=T_{2}^{*}(0)$,

$\kappa_{1} \frac{\partial T_{1}^{*}}{\partial y^{*}}=\kappa_{2} \frac{\partial T_{2}^{*}}{\partial y^{*}} \quad$ at $y^{*}=0$. 
Also, the boundary and the interface conditions on concentration for both the fluids are given by

$C_{1}^{*}(h)=C_{w_{1}}, \quad C_{2}^{*}(-h)=C_{w_{2}}$,

$C_{1}^{*}(0)=C_{2}^{*}(0)$,

$D_{1} \frac{\partial C_{1}^{*}}{\partial y^{*}}=D_{2} \frac{\partial C_{2}^{*}}{\partial y^{*}} \quad$ at $y^{*}=0$.

The continuity equations of both fluids imply that $v_{1}$ and $v_{2}$ are independent of $y$ and they can be at most function of time alone. Hence we can write

$v_{i}^{*}=v_{0}\left(1+\in A e^{i \omega t}\right), \quad \forall i=1,2$

where it is supposed that the transpiration velocity $v_{i}^{*}$ varies periodically with time about a non-zero constant mean $v_{0}$ and $\left(v_{1}=v_{2}\right), A$ is real positive constant and $\in$ small parameter such that $0<\in A \leq 1$. If $\in A=0$, the case of constant transpiration on velocity may be assumed.

\section{Method of Solution}

Introducing the following non-dimensional quantities:

$$
\begin{aligned}
& u_{i}=\frac{u_{i}^{*}}{U_{0}}, \quad y=\frac{y^{*}}{h}, \quad t=\frac{t^{*} v_{1}}{h^{2}}, \quad P=\frac{h^{2}}{\mu_{1} U}\left(\frac{\partial p^{*}}{\partial x^{*}}\right), \quad \theta_{i}=\frac{T_{i}^{*}-T_{w_{1}}^{*}}{T_{w_{2}}^{*}-T_{w_{1}}^{*}}, \quad S c=\frac{v_{1}}{D_{1}}, \quad D=\frac{D_{2}}{D_{1}}, \\
& C_{i}=\frac{C_{i}^{*}-C_{w_{1}}}{C_{w_{2}}-C_{w_{1}}}, \quad E c=\frac{U^{2}}{C p \Delta T}, \quad \eta=\frac{\rho_{1}}{\rho_{2}}, \quad m=\frac{\mu_{2}}{\mu_{1}}, \quad \operatorname{Pr}=\frac{\mu_{1} C p}{\kappa_{1}}, \quad \kappa=\frac{\kappa_{2}}{\kappa_{1}}, \\
& K_{p}^{* 2}=\frac{h^{2}}{K_{p}^{*}}, n=\frac{\beta_{c_{2}}}{\beta_{c_{1}}}, \quad v=\frac{v^{*}}{v_{0}}, \quad G c=\frac{\rho_{1} g h^{2} \beta_{c_{1}}\left(C_{w_{2}}^{*}-C_{w_{1}}^{*}\right)}{\mu_{1} U_{0}} .
\end{aligned}
$$

Where $i=1,2$ give the values for region I and II respectively.

Using (12) and (13) the governing equations from(1) to (8) are reduced in non-dimensional form as follows:

\section{Region-I (clear region)}

$$
\begin{aligned}
& \frac{\partial u_{1}}{\partial t}+\left(1+\in A e^{i \omega t}\right) \frac{\partial u_{1}}{\partial y}=\frac{\partial^{2} u_{1}}{\partial y^{2}}-P+G c C_{1}, \\
& \frac{\partial \theta_{1}}{\partial t}+\left(1+\in A e^{i \omega t}\right) \frac{\partial \theta_{1}}{\partial y}=\frac{1}{\operatorname{Pr}} \frac{\partial^{2} \theta_{1}}{\partial y^{2}}+E c\left(\frac{\partial u_{1}}{\partial y}\right)^{2}, \\
& \frac{\partial C_{1}}{\partial t}+\left(1+\in A e^{i \omega t}\right) \frac{\partial C_{1}}{\partial y}=\frac{1}{S c} \frac{\partial^{2} C_{1}}{\partial y^{2}},
\end{aligned}
$$

Region-II (porous region)

$$
\begin{aligned}
& \frac{\partial u_{2}}{\partial t}+\left(1+\in A e^{i \omega t}\right) \frac{\partial u_{2}}{\partial y}=m \frac{\partial^{2} u_{2}}{\partial y^{2}}-\eta P-K_{p} m \eta u_{2}+G c C_{2} n, \\
& \frac{\partial \theta_{2}}{\partial t}+\left(1+\in A e^{i \omega t}\right) \frac{\partial \theta_{2}}{\partial y}=\frac{1}{\operatorname{Pr}} \eta \frac{\partial^{2} \theta_{2}}{\partial y^{2}}+E c m\left(\frac{\partial u_{2}}{\partial y}\right)^{2}+m E c K_{p} u_{2}^{2},
\end{aligned}
$$




$$
\frac{\partial C_{2}}{\partial t}+v\left(1+\in A e^{i \omega t}\right) \frac{\partial C_{2}}{\partial y}=\frac{D}{S c} \frac{\partial^{2} C_{2}}{\partial y^{2}},
$$

The corresponding boundary conditions are reduced as

$$
\begin{aligned}
& u_{1}(1)=0, \quad u_{2}(-1)=0, \\
& u_{1}(0)=u_{2}(0), \\
& \frac{\partial u_{1}}{\partial y}=m \frac{\partial u_{2}}{\partial y} \quad \text { at } y=0 . \\
& \theta_{1}(1)=0, \quad \theta_{2}(-1)=1, \\
& \theta_{1}(0)=\theta_{2}(0), \\
& \frac{\partial \theta_{1}}{\partial y}=\kappa \frac{\partial \theta_{2}}{\partial y} \quad \text { at } y=0 .
\end{aligned}
$$

and

$C_{1}(1)=0, \quad C_{2}(-1)=1$

$C_{1}(0)=C_{2}(0)$

$\frac{\partial C_{1}}{\partial y}=D \frac{\partial C_{2}}{\partial y}$ at $y=0$

Equations (14) to (19) are coupled partial differential equations. They can be reduced to ordinary differential equation by assuming

$$
\begin{aligned}
& u_{i}(y, t)=u_{i 0}(y)+\in e^{i \omega t} u_{i 1}+O\left(\epsilon^{2}\right)+\cdots \cdot, i=1,2, \\
& \theta_{i}(y, t)=\theta_{i 0}(y)+\in e^{i \omega t} \theta_{i 1}+O\left(\epsilon^{2}\right)+\cdots \cdots, i=1,2, \\
& C_{i}(y, t)=C_{i 0}(y)+\in e^{i \omega t} C_{i 1}+O\left(\epsilon^{2}\right)+\cdots, i=1,2,
\end{aligned}
$$

where $i=1,2$ gives equations for region I and II respectively. This is a valid postulation becaus e we have assumed that the amplitude $\in A<<1$.

By substituting (23) to (25) into equations (14) to (19), equating the harmonic and non-harmonic terms, and neglecting the higher order terms of $\epsilon^{2}$, we get the following sets of differential equations:

\section{Region-I: Clear Region}

\section{Harmonic Terms}

$$
\begin{aligned}
& \frac{\partial^{2} u_{10}}{\partial y^{2}}-\frac{\partial u_{10}}{\partial y}=P-G c C_{10}, \\
& \frac{\partial^{2} \theta_{10}}{\partial y^{2}}-\operatorname{Pr} \frac{\partial \theta_{10}}{\partial y}=-E c \operatorname{Pr}\left(\frac{\partial u_{10}}{\partial y}\right)^{2}, \\
& \frac{\partial^{2} C_{10}}{\partial y^{2}}-S c \frac{\partial C_{10}}{\partial y}=0,
\end{aligned}
$$

\section{Non-Harmonic Terms}

$$
\frac{\partial^{2} u_{11}}{\partial y^{2}}-\frac{\partial u_{11}}{\partial y}-i \omega u_{11}=A \frac{\partial u_{10}}{\partial y}-G c C_{11} \text {, }
$$




$$
\begin{aligned}
& \frac{\partial^{2} \theta_{11}}{\partial y^{2}}-\operatorname{Pr} \frac{\partial \theta_{11}}{\partial y}-i \omega \operatorname{Pr} \theta_{11}=-2 \operatorname{Pr}\left(\frac{\partial u_{10}}{\partial y}\right)\left(\frac{\partial u_{11}}{\partial y}\right)-A \operatorname{Pr} \frac{\partial \theta_{10}}{\partial y}, \\
& \frac{\partial^{2} C_{11}}{\partial y^{2}}-S c \frac{\partial C_{11}}{\partial y}-S c i \omega \frac{\partial C_{11}}{\partial y}=A S c \frac{\partial C_{10}}{\partial y} .
\end{aligned}
$$

\section{Region-II: Porous Region}

\section{Harmonic Terms}

$m \frac{\partial^{2} u_{20}}{\partial y^{2}}-\frac{\partial u_{20}}{\partial y}-m^{2} K_{p} \eta u_{20}=P-G \operatorname{cn} C_{20}$,

$\frac{\eta}{\operatorname{Pr}} \frac{\partial^{2} \theta_{20}}{\partial y^{2}}-\frac{\partial \theta_{20}}{\partial y}=-m E c\left(\frac{\partial u_{20}}{\partial y}\right)^{2}-m E c K_{p} u_{20}^{2}$,

$\frac{\partial^{2} C_{20}}{\partial y^{2}}-\frac{S c}{D} \frac{\partial C_{20}}{\partial y}=0$

\section{Non-Harmonic Terms}

$m \frac{\partial^{2} u_{21}}{\partial y^{2}}-\frac{\partial u_{21}}{\partial y}-\left(m K_{p} \eta+i \omega\right) u_{21}=A \frac{\partial u_{20}}{\partial y}-G c C_{21} n$

$\frac{\eta}{\operatorname{Pr}} \frac{\partial^{2} \theta_{21}}{\partial y^{2}}-\frac{\partial \theta_{21}}{\partial y}-i \omega \theta_{21}=-A\left(\frac{\partial \theta_{20}}{\partial y}\right)-2 m E c \frac{\partial u_{20}}{\partial y} \frac{\partial u_{21}}{\partial y}-2 m E c K_{p} u_{20} u_{21}$,

$\frac{\partial^{2} C_{21}}{\partial y^{2}}-\frac{S c}{D} \frac{\partial C_{21}}{\partial y}-\frac{i \omega S c}{D} C_{21}=A \frac{S c}{D} \frac{\partial C_{20}}{\partial y}$,

The corresponding boundary and interface conditions are

$$
\begin{aligned}
& u_{1 i}(1)=0, \quad u_{2 i}(-1)=0, \\
& u_{1 i}(0)=u_{2 i}(0), \\
& \frac{\partial u_{1 i}}{\partial y}=m \frac{\partial u_{2 i}}{\partial y} \text { at } y=0 . \\
& \theta_{1 i}(1)=0, \quad \theta_{2 i}(-1)=1, \\
& \theta_{1 i}(0)=\theta_{2 i}(0), \\
& \frac{\partial \theta_{1 i}}{\partial y}=\kappa \frac{\partial \theta_{2 i}}{\partial y} \text { at } y=0 . \\
& C_{1 i}(1)=0, \quad C_{2 i}(-1)=1, \\
& C_{1 i}(0)=C_{2 i}(0), \\
& \frac{\partial C_{1 i}}{\partial y}=D \frac{\partial C_{2 i}}{\partial y} \text { at } y=0 .
\end{aligned}
$$

where $i=0,1$ gives the boundary and interface conditions for harmonic and non-harmonic coefficients, respectively.

Now, the equations (26) to (37) are ordinary linear coupled differential equations with constant coefficients. These are solvable by the straight forward calculation. 


\section{Skin-Friction Coefficient}

The non-dimensional shearing stress in terms of coefficient of skin-friction at the upper plate $C_{f}(u)$ and lower plate $C_{f}(l)$ are given by

$$
\begin{aligned}
& C_{f}(u)=\frac{1}{\operatorname{Re}}\left[\left(\frac{\partial u_{10}}{\partial y}\right)_{y=1}+\in e^{i \omega t}\left(\frac{\partial u_{11}}{\partial y}\right)_{y=1}\right], \\
& C_{f}(u)=\frac{1}{\operatorname{Re}}\left[\left(\frac{\partial u_{10}}{\partial y}\right)_{y=1}+\in e^{i \omega t}\left(\frac{\partial u_{11}}{\partial y}\right)_{y=1}\right],
\end{aligned}
$$

\section{Nusselt Number}

The rate of heat transfer in terms of Nusselt number at the upperplate $N u(u)$ and lower plate $N u(l)$ are given by

$$
\begin{aligned}
& N u(u)=-\left[\left(\frac{\partial \theta_{10}}{\partial y}\right)_{y=1}+\in e^{i \omega t}\left(\frac{\partial \theta_{11}}{\partial y}\right)_{y=1}\right], \\
& N u(l)=-\left[\left(\frac{\partial \theta_{20}}{\partial y}\right)_{y=-1}+\in e^{i \omega t}\left(\frac{\partial \theta_{21}}{\partial y}\right)_{y=-1}\right],
\end{aligned}
$$

\section{Sherwood Number}

The rate of mass transfer in term of Sherwood number at the upper plate $S h(u)$ and lower plate $S h(l)$ are given by

$$
\begin{aligned}
& S h(u)=-\left[\left(\frac{\partial C_{10}}{\partial y}\right)_{y=1}+\in e^{i \omega t}\left(\frac{\partial C_{11}}{\partial y}\right)_{y=1}\right], \\
& \operatorname{Sh}(l)=-\left[\left(\frac{\partial C_{20}}{\partial y}\right)_{y=-1}+\in e^{i \omega t}\left(\frac{\partial C_{21}}{\partial y}\right)_{y=-1}\right],
\end{aligned}
$$

\section{Results and Discussions}

The present study is focused on the unsteady oscillatory flow and heat \& mass transfer in a horizontal channel partially occupied by porous medium one of them is filled by porous material and other one is clear region. In the paper, the effects of the dimensionless parameters on the velocity, temperature and concentration distribution for both the regions are discussed by assigning numerical values to these parameters. Separate solutions for each fluid region are obtained and these solutions are matched at the interface conditions using suitable matching conditions. The results are depicted graphically in figures from (2) to (14).

Fig. 2 exhibits the velocity profile in the presence of different porous medium parameter. As the porous medium parameter Kp increases, the fluid velocity decreases at all the points in both the region of the channel. This is expected due to the interference in the flow created by the porous matrix in region-II. This is also interesting that the value of the velocity field reaches at high peak point in region-II comparative to region-I and then approaches to the boundary conditions and matching the interface condition of both the regions.

This solutions is also similar to the results of the problem considered in Umavathi et al.(2010).

Fig. 3 shows the increase in the mass buoyancy leads to decrease the velocity of the fluid in both the regions. It is found that the fluid velocity rises from the prescribed value at $y=1$ on upper plate of the channel, attains a maximum value and then falls gradually to zero as boundary condition on lower plate of the channel. Fig. 4 
shows that when diffusing species become more heavier with destructive reaction, then the fluid velocity reduces throughout the flow between the horizontal plates.

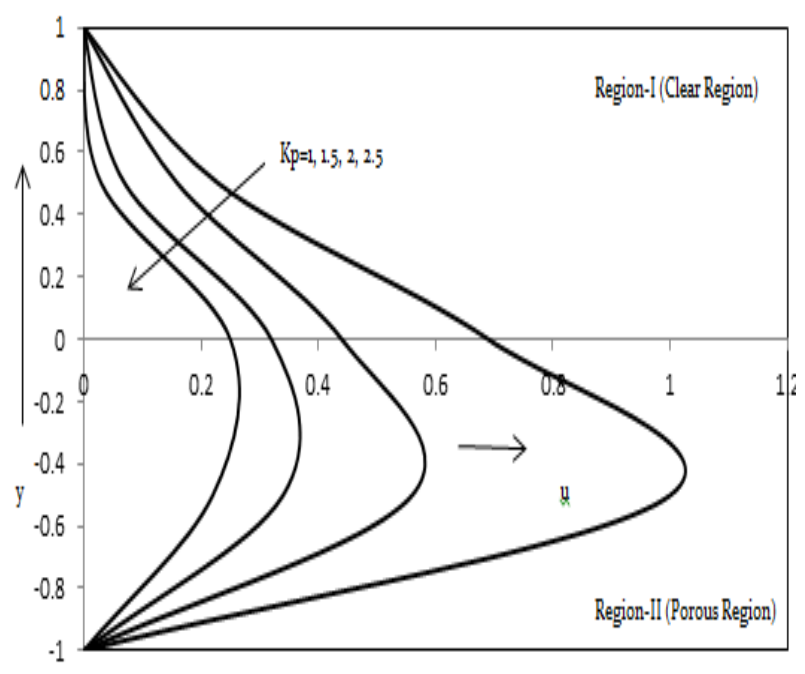

Fig. 2: Velocity distribution versus y when and $\mathrm{Gc}=3$, $\mathrm{D}=1, \mathrm{~m}=1, \mathrm{Sc}=0.22, \mathrm{P}=1, \mathrm{k}=1, \mathrm{Pr}=0.71$ and $\mathrm{\eta}=1$.

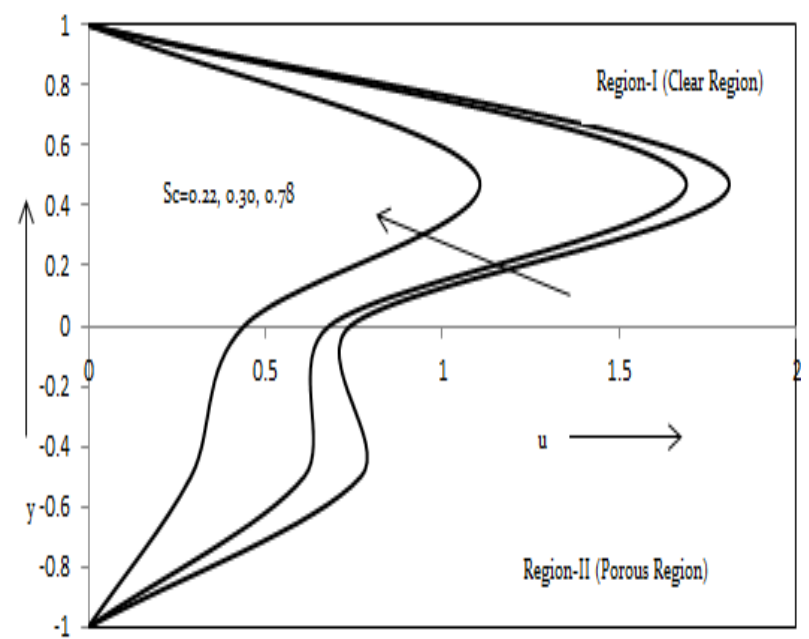

Fig. 4: Velocity distribution versus y when $\operatorname{Pr}=0.71$, $\mathrm{Gc}=3, \mathrm{D}=1, \mathrm{~m}=1, \omega=5, \mathrm{P}=1, \mathrm{k}=1, \mathrm{~K} p=1$ and $\eta=1$.

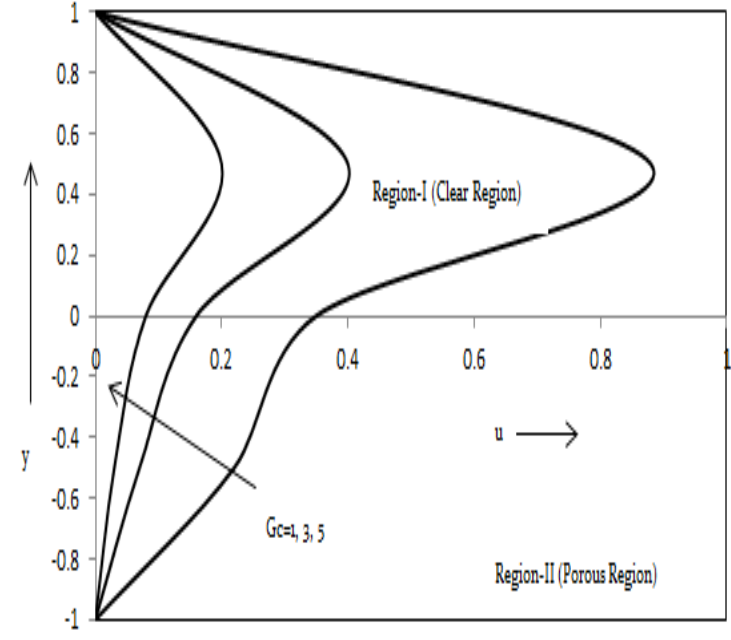

Fig. 3: Velocity distribution versus y when $\mathrm{P}=1$, $\kappa=1, \mathrm{Kp}=1, \quad \operatorname{Pr}=0.71, \quad \mathrm{Sc}=0.22, \quad \mathrm{D}=1, \quad \mathrm{~m}=1, \omega=1 \quad$ and $\eta=1$.

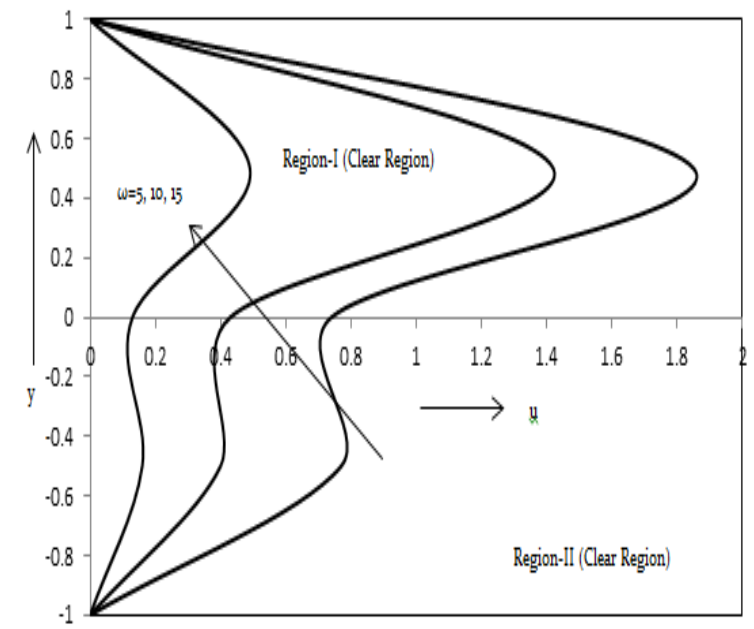

Fig. 5: Velocity distribution versus y when $G c=3, D=1$, $\mathrm{m}=1, \mathrm{Sc}=0.22, \mathrm{P}=1, \mathrm{k}=1, \mathrm{Kp}=1, \operatorname{Pr}=0.71$ and $\mathrm{\eta}=1$.

Fig. 5 represents the effect of frequency parameters $\omega$ on velocity field. It is observed that the fluid velocity decreases with the increases of frequency parameter, individually in both the region of the channel. It is noted that the magnitude of promotion is large in region-I(clear region) comparative to region-II (porous region).

Fig. 6 exhibits the effect of viscosity ratio $m$ on the velocity of the fluid. It can be seen that, with an increase in viscosity ratio the fluid velocity decreases. This is due to the fact that when the fluid viscosity increases, then the fluid in both the region of the channel become thicker and hence the velocity of the fluid is reduced. Fig. 7 displays the influence of molecular diffusivity ratio in velocity field. An increase in the molecular diffusivity ratio, decreases the fluid velocity in both the regions. But this effect is more prominent in region-II of porous matrix. 
P. Sharma and R. Saboo/ Journal of Naval Architecture and Marine Engineering, 14(2017) 101-114

Fig. 8 represents the variation of temperature field with porous medium parameter Kp. In the presence of constant viscosity parameter, as the porous medium parameter $\mathrm{Kp}$ increases, temperature decreases in both the regions of the channel. This is the same effect as in velocity field.

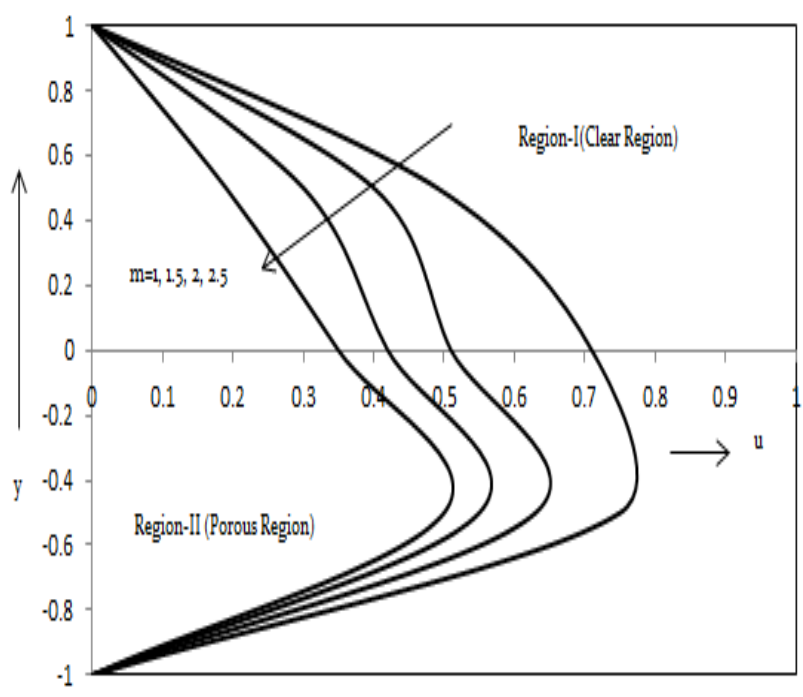

Fig. 6: Velocity distribution versus y when $\mathrm{Gc}=3, \mathrm{D}=1$, $\mathrm{Sc}=0.22, \omega=5, \mathrm{P}=1, \mathrm{Kp}=1, \operatorname{Pr}=0.71$ and $\eta=1$.

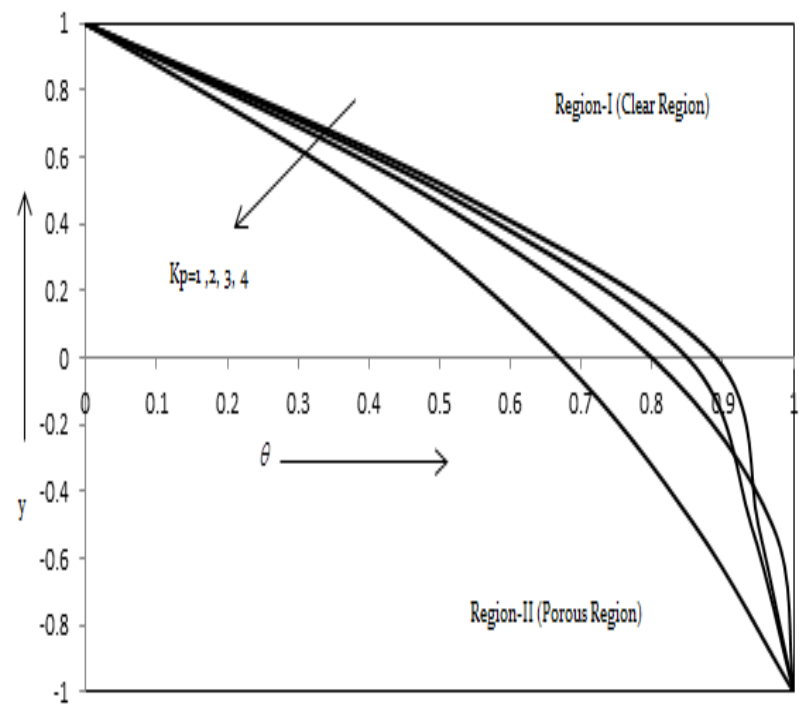

Fig. 8: Temperature distribution versus y when $\mathrm{Gc}=3$, $\mathrm{D}=1, \mathrm{~m}=1, \omega=5, \mathrm{P}=1, \mathrm{Ec}=1, \mathrm{k}=1, \mathrm{Pr}=0.71, \mathrm{Sc}=0.22$ and $\eta=1$

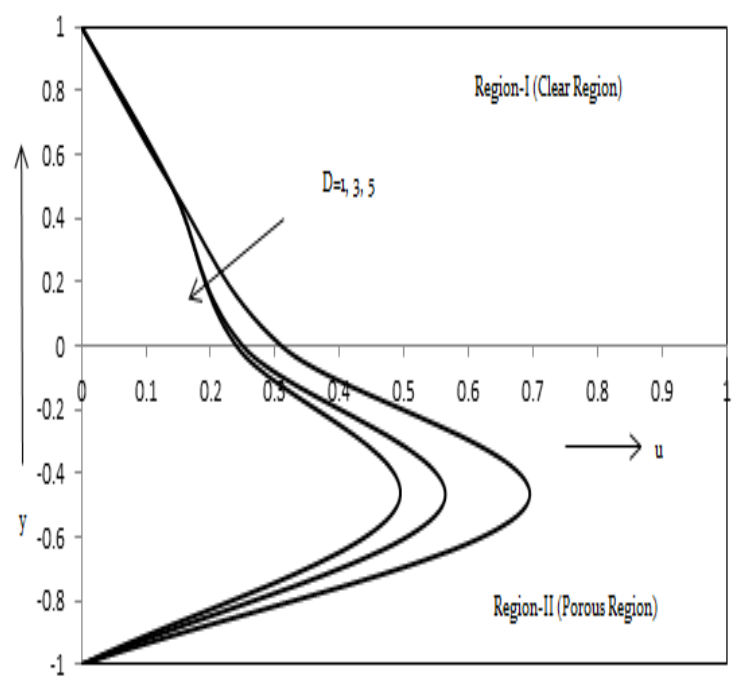

Fig. 7: Velocity distribution versus y when $\operatorname{Pr}=0.71$, $\mathrm{Gc}=3, \mathrm{Sc}=0.22, \omega=5, \mathrm{P}=1, \mathrm{Kp}=1$ and $\eta=1$.

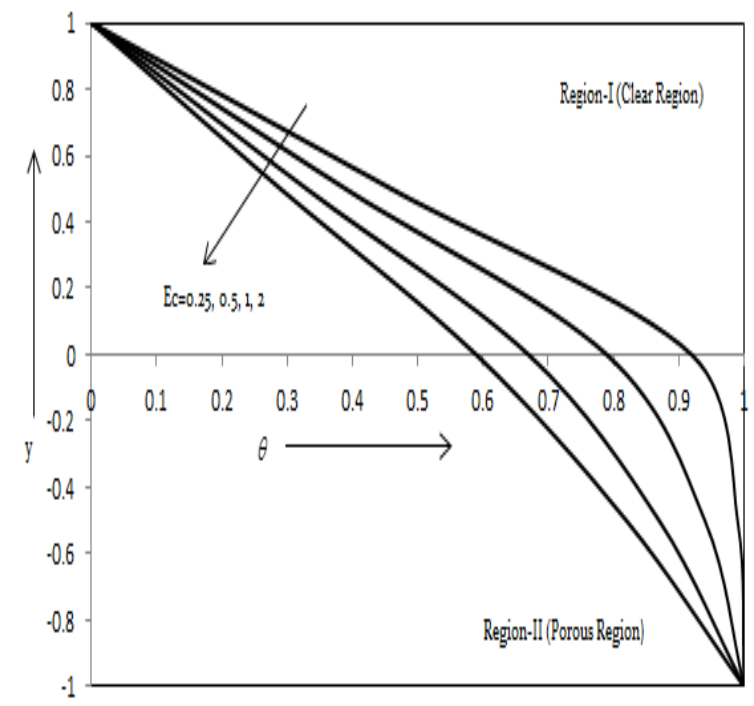

Fig. 9: Temperature distribution versus $y$ when $\mathrm{Gc}=3, \mathrm{D}=1, \mathrm{~m}=1, \omega=5, \mathrm{P}=1, \mathrm{Kp}=1, \mathrm{k}=1, \mathrm{Pr}=0.71$, Sc $=0.22$ and $\eta=1$.

This is depicted from Fig. 9 that due to the increase in Eckert number the fluid temperature decreases at all the points of both regions of the channel. This result is also expected as the effect of viscous and porous medium dissipation increases with the increase of Eckert number. Therefore the magnitude of promotion in fluid temperature is large in region-II.

Fig. 10 displays the influence of viscosity ratio on the temperature profiles. An increase in the viscosity ratio, decreases the fluid temperature in both the regions due to the increase of thickness of the fluid in both the regions. Fig. 11 shows the effect of the thermal conductivity ratio $\kappa$ on the temperature field. It is noticed that an increment in the thermal conductivity ratio $\kappa$ reduces the value of the temperature field at all the points of both the regions. Because increase in the thermal conductivity ratio $\kappa$ have the tendency to cool down the thermal state in the channel. 
The effect of Prandtl number Pr on the temperature field is showed from Fig. 12. The fluid temperature increases with the increase of Prandtl number due to the increase of the thickness of thermal boundary layer. Variation of concentration in flow domain depicted through Figs. 13 and 14. From Fig. 13, it is noticed that fluid concentration props up with the high values of Sc [heavier species with low diffusivity, $\mathrm{Sc}=0.22$ (Hydrogen), $\mathrm{Sc}=0.78$ (Amonia) and $\mathrm{Sc}=2.62$ (Propyl benzene)]. Since destructive Schmidt number retards the thickness of solutal boundary layer. Because of that mass transfer takes its high values.

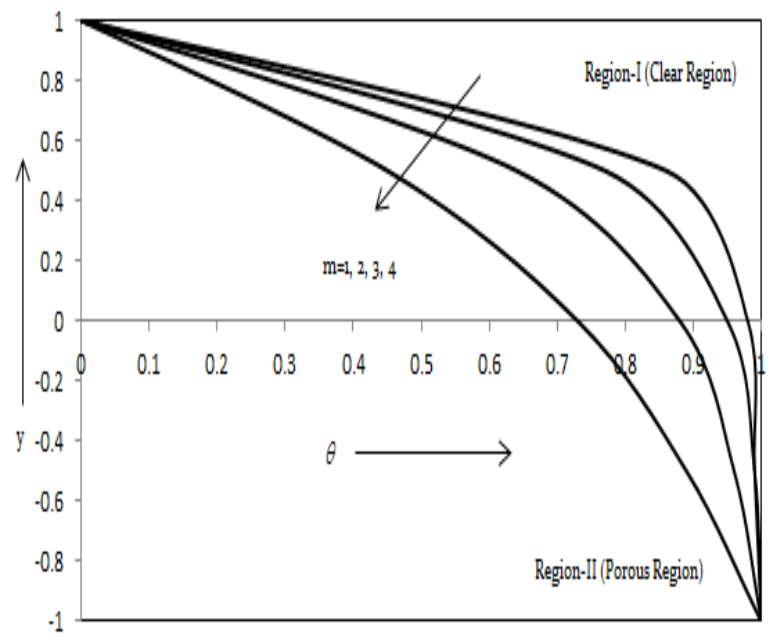

Fig. 10: Temperature distribution versus $y$ when $\mathrm{Gc}=3, \mathrm{D}=1, \mathrm{Ec}=1 \mathrm{\omega}=5, \mathrm{P}=1, \mathrm{Pr}=0.71, \mathrm{k}=1, \mathrm{Sc}=0.22$ and $\eta=1$.

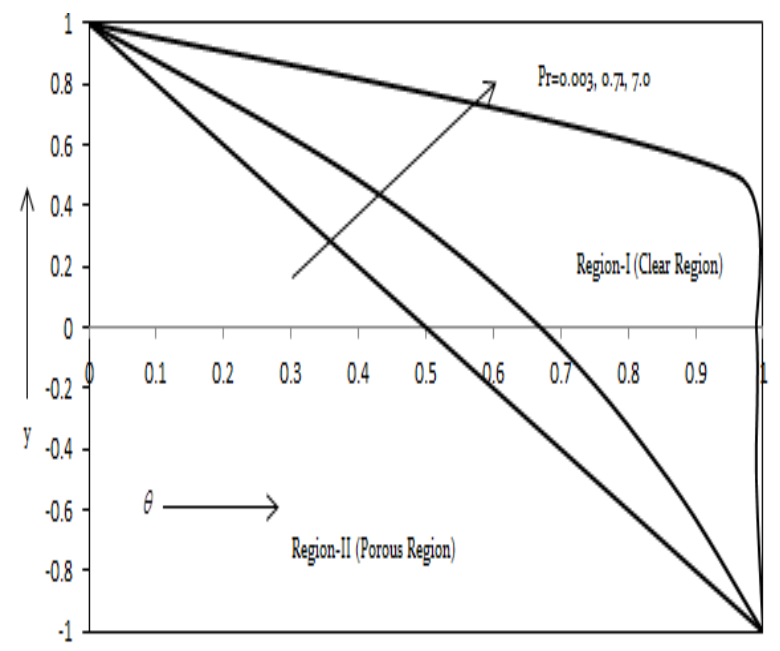

Fig. 12: Temperature distribution versus $y$ when $\mathrm{Gc}=3, \mathrm{D}=1, \mathrm{~m}=1, \omega=5, \mathrm{P}=1, \mathrm{Ec}=1, \mathrm{Kp}=1, \mathrm{k}=1, \mathrm{Sc}=0.22$ and $\eta=1$

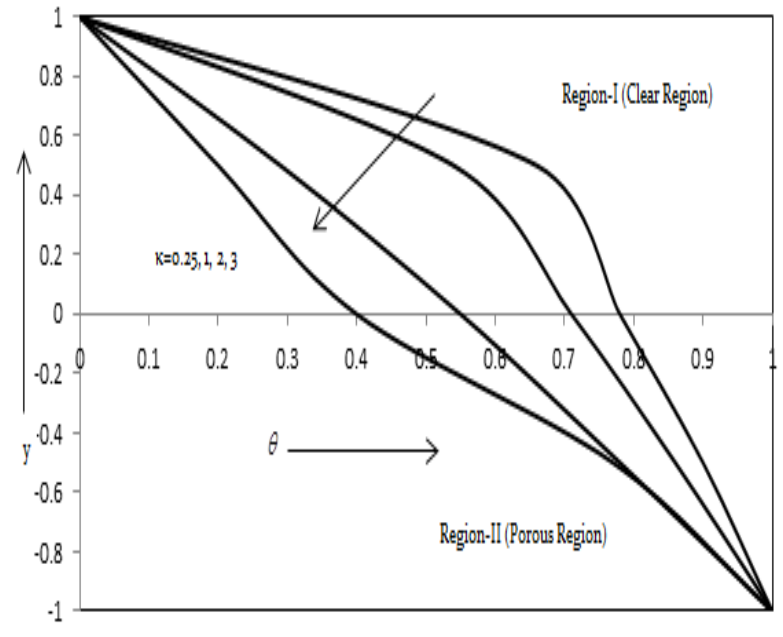

Fig. 11: Temperature distribution versus y when $\mathrm{Gc}=3$, $D=1, m=1, \omega=5, P=1, E c=1, K p=1, \operatorname{Pr}=0.71, S c=0.22$ and $\eta=1$.

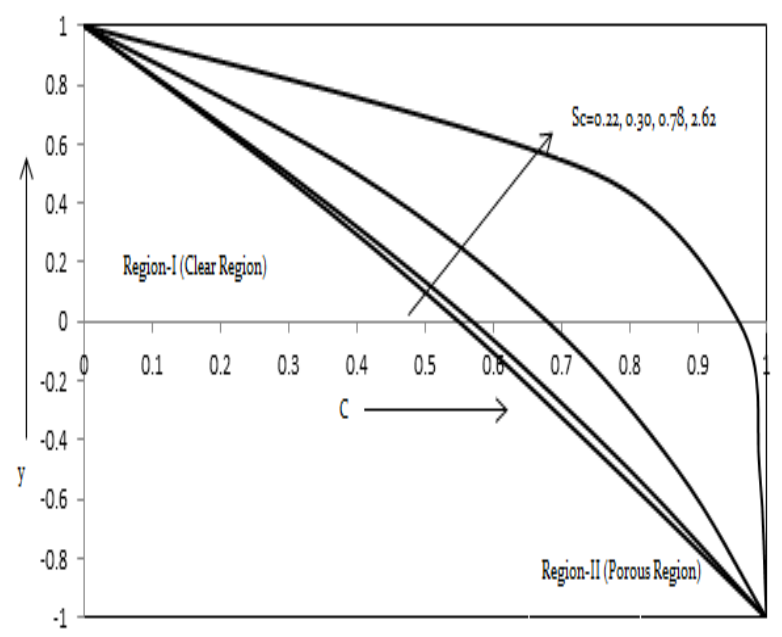

Fig. 13: Concentration distribution versus $\mathrm{y}$ when $\mathrm{Gc}=3, \mathrm{D}=1, \mathrm{~m}=1, \omega=5, \mathrm{P}=1, \kappa=1, \mathrm{Ec}=1, \mathrm{Kp}=1, \mathrm{Pr}=0.71$ and $\eta=1$

In Fig. 14, the effect of the diffusivity ratio D on the fluid concentration is presented. It is observed that increments in the diffusivity ratio props up the magnitude of the fluid concentration.

Skin-friction, Nusselt number and Sherwood number provides flow characteristic at the boundary surface which is significant for thermal and solutal stability of flow. Table 1 shows the coefficient of skin-friction at upper plate increases with an increase of frequency parameter and diffusivity ratio; while decreases with the increases 
P. Sharma and R. Saboo/ Journal of Naval Architecture and Marine Engineering, 14(2017) 101-114 of Schmidt number, mass buoyancy, viscosity ratio and porous medium parameter. The coefficient of skin friction at lower plate increases with an increase of frequency parameter; while decreases with an increases of Schmidt number, mass buoyancy, porosity parameter and diffusivity ratio. This is noticeable that the effect of pressure gradient is quite negligible on skin-friction coefficient at both the plates.

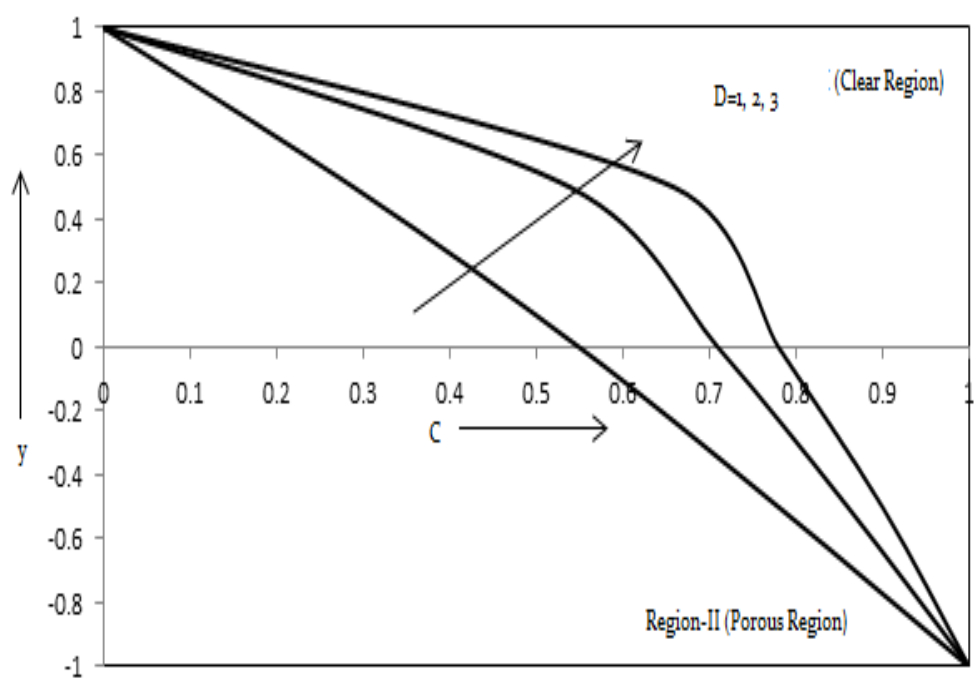

Fig. 14: Concentration distribution versus y when $\mathrm{Gc}=3, \mathrm{~m}=1, \omega=5, \mathrm{P}=1, \mathrm{Ec}=1, \mathrm{Kp}=1, \mathrm{Sc}=0.22, \operatorname{Pr}=0.71, \mathrm{k}=1$ and $\eta=1$.

This is observed from the Table 2 that Nusselt number at the upper plate props up with the increase of frequency parameter while its decrease with all others parameters. Nusselt number at lower plate rises with the increases of Prandtl number, Eckert number, Schmidt number, thermal conductivity, mass buoyancy, viscosity ratio, Hartman number and porosity parameter; while decreases with pressure gradient, frequency parameter and molecular diffusivity.

Table 1: The numerical values of coefficient of Skin-Friction at the upper and lower plate of the channel for different values of physical parameters when $\in=0.025, \operatorname{Re}=1.00$ and $\omega t=\pi / 2$.

\begin{tabular}{|l|l|l|l|l|l|l|l|l|l|}
\hline S. No. & $S c$ & $G c$ & $m$ & $P$ & $\omega$ & $K_{p}$ & $\mathrm{D}$ & $C_{f}(u)$ & $C_{f}(l)$ \\
\hline I & 0.22 & 5 & 1 & 1 & 5 & 1 & 1 & 25.011 & 41.326 \\
\hline II & 0.30 & 5 & 1 & 1 & 5 & 1 & 1 & 16.038 & 25.226 \\
\hline III & 0.78 & 5 & 1 & 1 & 5 & 1 & 1 & 4.062 & 5.710 \\
\hline IV & 2.62 & 5 & 1 & 1 & 5 & 1 & 1 & -2.466 & 0.8733 \\
\hline V & 0.22 & 10 & 1 & 1 & 5 & 1 & 1 & -45.61 & 0.1042 \\
\hline VI & 0.22 & 5 & 2 & 1 & 5 & 1 & 1 & -15.033 & -74.25 \\
\hline VII & 0.22 & 5 & 1 & 2 & 5 & 1 & 1 & 24.991 & 41.32 \\
\hline VIII & 0.22 & 5 & 1 & 1 & 10 & 1 & 1 & 49.982 & 82.65 \\
\hline IX & 0.22 & 5 & 1 & 1 & 5 & 2 & 1 & -0.8466 & 1.331 \\
\hline X & 0.22 & 5 & 1 & 1 & 5 & 1 & 2 & 40.36 & -1.14 \\
\hline
\end{tabular}

Table 3 shows that the Sherwood number at upper plate rises with an increases of Schmdit number and diffusivity ratio while adverse behaviour is observed at the case of lower plate. 
P. Sharma and R. Saboo/ Journal of Naval Architecture and Marine Engineering, 14(2017) 101-114 Table 2: The numerical values of Nusselt number at upper and lower plate of the channel for different values physical parameters when $\in=0.025$ and $\omega t=\pi / 2$.

\begin{tabular}{|l|l|l|l|l|l|l|l|l|l|l|l|l|}
\hline S. No. & Pr & Ec & Sc & $\kappa$ & Gc & M & P & $\omega$ & $\mathrm{Kp}$ & $\mathrm{D}$ & $\mathrm{Nu}(\mathrm{u})$ & $\mathrm{Nu}(\mathrm{l})$ \\
\hline I & 0.71 & 0.5 & 0.22 & 2 & 5 & 1 & 1 & 5 & 1 & 1 & -0.625 & -0.392 \\
\hline II & 7.0 & 0.5 & 0.22 & 2 & 5 & 1 & 1 & 5 & 1 & 1 & -0.705 & -0.343 \\
\hline III & 0.71 & 1 & 0.22 & 2 & 5 & 1 & 1 & 5 & 1 & 1 & -0.629 & -0.390 \\
\hline IV & 0.71 & 0.5 & 0.30 & 2 & 5 & 1 & 1 & 5 & 1 & 1 & -0.677 & -0.352 \\
\hline V & 0.71 & 0.5 & 0.78 & 2 & 5 & 1 & 1 & 5 & 1 & 1 & -0.999 & -0.077 \\
\hline VI & 0.71 & 0.5 & 0.22 & 4 & 5 & 1 & 1 & 5 & 1 & 1 & -0.663 & -0.367 \\
\hline VII & 0.71 & 0.5 & 0.22 & 2 & 10 & 1 & 1 & 5 & 1 & 1 & -0.759 & -0.337 \\
\hline VIII & 0.71 & 0.5 & 0.22 & 2 & 5 & 2 & 1 & 5 & 1 & 1 & -0.640 & -0.383 \\
\hline IX & 0.71 & 0.5 & 0.22 & 2 & 5 & 1 & 2 & 5 & 1 & 1 & -0.853 & -0.608 \\
\hline X & 0.71 & 0.5 & 0.22 & 2 & 5 & 1 & 1 & 10 & 1 & 1 & -0.618 & -0.397 \\
\hline XI & 0.71 & 0.5 & 0.22 & 2 & 5 & 1 & 1 & 5 & 3 & 1 & -0.651 & -0.375 \\
\hline XII & 0.71 & 0.5 & 0.22 & 2 & 5 & 1 & 1 & 5 & 1 & 3 & -0.804 & -0.505 \\
\hline
\end{tabular}

Table 3: The numerical values of Sherwood number at lower and upper plate of the channel for different values of physical parameters when $\in=0.025$ and $\omega t=\pi / 2$.

\begin{tabular}{|l|l|l|l|l|}
\hline S. No. & $S c$ & $D$ & $S h(u)$ & $S h(l)$ \\
\hline I & 0.22 & 2 & 0.7826 & 0.5040 \\
\hline II & 0.30 & 2 & 0.8278 & 0.4543 \\
\hline III & 0.78 & 2 & 1.1310 & 0.2376 \\
\hline IV & 2.62 & 2 & 2.6725 & 0.0141 \\
\hline V & 0.22 & 4 & 0.7963 & 0.0377 \\
\hline
\end{tabular}

\section{Conclusions}

In the present paper we considered the unsteady oscillatory flow of viscous incompressible fluid with heat and mass transfer in a horizontal channel partially filled with porous medium. It is concluded that:

I. Presence of porous medium offers a resistance to velocity and temperature distribution. Therefore velocity and temperature field can be controlled by the density of porous matrix.

II. Velocity profile decreases as the porous medium parameter Kp increases, due to the interference in the flow created by the porous matrix in region-II.

III. The fluid temperature amplifies with the ascendency of inertial forces over viscous forces. Due to that fluid temperature is more for water in compression of air.

IV. Prandtl number boosts up the fluid temperature due to the upsurge of the thickness of thermal boundary layer.

V. Fluid concentration props up for the heavier diffusive species and high molecular diffusivity ratio because destructive chemical retards the thickness of solutal boundary layer.

\section{References}

Adegun, I., Komolafe, O., Hussein, A. and Oyekale, J. (2014): A 3D finite element analysis of incompressible fluid flow and contaminant transport through a porous landfill, Journal of Engineering Science and Technology, Vol. 9(4), pp. 477-489.

Ahmed, S. E., Hussein, A. K., Mohammed, H. A., Adegun, I. K., Zhang, X., Kolsi, L., Hasanpour, A. and Sivasankaran, S. (2014): Viscous dissipation and radiation effects on MHD natural convection in a square enclosure filled with a porous medium, Nuclear Engineering and Design, Vol. 266, pp. 34-42. 
Bakkas, M., Amahmid, A. and Hasnaoui, M. (2008): Numerical study of natural convection heat transfer in a horizontal channel provide with rectangular blocks releasing uniform heat flux and mounted on its lower wall, Energy Conversion and Management, Vol. 49, pp. 2757-2766.

http://dx.doi.org/10.1016/j.enconman.2008.03.017

Chen, G. M. and Tso, C. P. (2011): Forced convection with viscous dissipation using a two -equation model in a channel filled by a porous medium, Int. J. of Heat and Mass Transfer, Vol.54, pp.1791-1804. http://dx.doi.org/10.1016/j.ijheat masstransfer.2010.12.037

Ghachem, K., Kolsi, L., Maatki, C., Hussein, A. and Borjini, M. (2012): Numerical simulation of threedimensional double diffusive free convection flow and irreversibility studies in a solar distiller, Int. Comm. in Heat and Mass Transfer, Vol. 39, pp. 869-876. https://dx.doi.org/10.1016/j.icheatmasstransfer.2012.04.010

Hussein, A. K. and Hussain, S. (2013): Natural convection in a square enclosure filled with a saturated porous matrix under different discrete heat sources locations, World Academy of Science, Engineering and Technology, Vol. 78, pp. 1896-1902.

Kuznestov, A. V. (2001): Influence of thermal dispersion on forced convection in a composite parallel plate channel, ZAMP J. Applied Mathematics Physics, Vol. 52, pp. 135-150. https://dx.doi.org/10.1007/PL00001536

Kuznetsov, A. V. and Nield, D. A. (2010): Forced convection in a channel partly occupied by bidis perse porous medium asymmetric case, Int. J. of Heat and Mass Transfer, Vol. 53 pp. 5167-5175. https://dx.doi.org/10.1016/j.ijheatmass transfer.2010.07.046

Kumar, N., Jain, T. and Gupta, S. (2012): Unsteady MHD free convective flow through porous medium sandwiched between viscous fluid, Int. J. of Energy \& Technology, Vol.27, No.4, pp.1-11.

Maatki, C., Ghachem, K., Kolsi, L., Hussein, A., Borjini, M. and Ben Aissia, H. (2016): Inclination effects of magnetic field direction in 3D-double-diffusive natural convection, Appl. Math. And Comp., Vol. 273, pp. 178189. https://dx.doi.org/10.1016/j.amc.2015.09.043

Matin, M. H. and Pop, I. (2013): Forced convection heat and mass transfer flow of a nanofluid through a porous channel with a first order chemical reaction on the wall, Int. Communication in Heat and Mass Transfer, Vol. 46, pp. 134-141. https://dx.doi.org/10.1016/j.icheatmas stransfer.2013.05.001

Nield, D. A., Kuznetsov, A. V. and Xiong, M. (2004): Effects of viscous dissipation and flow work on forced convection in a channel filled by saturated porous medium, Transport in Porous Media, Vol. 56, pp. 351-367. https://dx.doi.org/10.1023/B:TIPM.0000026087.77213.c8

Raju, K.V. S., Reddy, T. S., Raju, M. C., Narayana, P. V. S. and Venkataramana, S. (2014): MHD convective flow through porous medium in a horizontal channel with insulated and impermeable bottom wall in the presence of viscous dissipation and joule heating, Ain Shams Engineering Journal, Vol. 5, pp. 543-551. https://dx.doi.org/10.1016/j.asej.2103.12.2014

Reddy Ramana, J. V., Sugunamma, V. and Sandeep, N. (2017) Dual solutions for heat and mass transfer in chemically reacting radiative non-newtonian fluid with aligned magnetic field, Journal of Naval Architecture and Marine Engineering, Vol. 14, pp. 25-38.

https://dx.doi.org/10.3329/jname.v14i1.25907

Sharma P. and Saboo R. (2017): A theoretical study of heat and mass transfer in forced convective chemically reacting radiating MHD flow through saturated porous medium over fixed horizontal channel, AMSE J.-AMSE IIETA, Vol. 78, pp. 100-115.

Sharma P. R., Kumar, N. and Sharma P. (2011): Influence of chemical reaction and radiation on unsteady MHD free convective flow and mass transfer through viscous incompressible fluid past a heated vertical plate immersed in porous medium in the presence of heat source, Applied Mathematical Science, Vol. 5, pp. 22492260.

Umavathi, J. C., Chamkha, A. J., Mateen, A. and Al-Mudhal, A. (2005): Unsteady two fluids flow and heat transfer in a horizontal channel, Heat and Mass transfer, Vol. 42, pp. 81-90.

Umavathi, J. C., Chamkha, A. J., Mateen, A. and Al-Mudhal, A. (2009): Unsteady oscillatory flow and heat transfer in a horizontal composite porous medium channel, Modelling and Control, Vol. 14, pp. 397-415. 